\title{
Psychometric Properties of Multi-Dimensional Scale of Perceived Social Support in Chinese Parents of Children with Cerebral Palsy
}

\author{
Yongli Wang ${ }^{1}$, Qin Wan ${ }^{1}$, Zhaoming Huang ${ }^{1 *}$, Li Huang ${ }^{2}$ and Feng Kong ${ }^{3 *}$ \\ 'Education and Rehabilitation Department, Faculty of Education, East China Normal University, Shanghai, China, \\ ${ }^{2}$ Rehabilitation Department, Yueyang Hospital of Integrated Traditional Chinese and Western Medicine, Shanghai University \\ of Traditional Chinese Medicine, Shanghai, China, ${ }^{3}$ School of Psychology, Shanxi Normal University, Xi'an, China
}

OPEN ACCESS

Edited by:

Livio Provenzi,

Eugenio Medea (IRCCS), Italy

Reviewed by:

Mitho Müller,

Ludwig-Maximilians-Universität

München, Germany

Olena Chorna

Fondazione Stella Maris (IRCCS), Italy

${ }^{*}$ Correspondence:

Feng Kong

kongfeng87@126.com; kongfeng@snnu.edu.cn

Zhaoming Huang

zhaoming_huang@hotmail.com

Specialty section:

This article was submitted to

Quantitative Psychology

and Measurement,

a section of the journal

Frontiers in Psychology

Received: 19 September 2017 Accepted: 06 November 2017

Published: 21 November 2017

Citation:

Wang Y, Wan Q, Huang Z, Huang L and Kong F (2017) Psychometric

Properties of Multi-Dimensional Scale

of Perceived Social Support

in Chinese Parents of Children with

Cerebral Palsy.

Front. Psychol. 8:2020.

doi: 10.3389/fpsyg.2017.02020
The Multi-dimensional Scale of Perceived Social Support (MSPSS) is one of the most extensively used instruments to assess social support. The purpose of this research was to test the reliability, factorial validity, concurrent validity and measurement invariance across gender groups of the MSPSS in Chinese parents of children with cerebral palsy. A total of 487 participants aged 21-55 years were recruited to complete the Chinese MSPSS and Parenting Stress Index-Short Form (PSI-SF). Composite reliability was calculated as the internal consistency of the Chinese MSPSS and a (multi-group) confirmatory factor analysis (CFA) was conducted to test the factorial validity and measurement invariance across gender. And Pearson correlations were calculated to test the relationships between MSPSS and PSI-SF. The Chinese MSPSS had satisfactory internal reliability with composite reliability values of more than 0.7 . The CFA indicated that the original three-factor model was replicated in this specific population. Importantly, the results of the multi-group CFA demonstrated that configural, metric, and scalar invariance across gender groups was supported. In addition, all the three subscales of MSPSS were significant related with PSI-SF. These findings suggest that the Chinese MSPSS is a reliable and valid tool for assessing social support and can generally be utilized across sex in the parents of children with cerebral palsy.

Keywords: social support, confirmatory factor analysis, validity, reliability, measurement invariance

\section{INTRODUCTION}

Cerebral palsy (CP), as a common cause of disability in children, is a serious threat to parents' psychological and physical health (Cheshire et al., 2010; Whittingham et al., 2013). Social support is an important resource to cope with various stressors (Campbell et al., 2011), and can mediate the influence of parenting stress on psychological and physical health in parents of children with CP (Jeong et al., 2013). In this study, we sought to validate a social support scale in a sample of Chinese parents of children with $\mathrm{CP}$, because specific population groups may have different social support expectations, social networks, and cultural backgrounds than the normal adolescents, verification of the reliability and validity of assessment scales in different special groups is very necessary and useful (Kim et al., 2008).

Social support is a multidimensional construct, and the types of social support vary widely (Stanley et al., 1998). In numerous social support instruments, Multi-dimensional Scale of Perceived Social Support (MSPSS) is a considered ideal assessment tool 
(Bruwer et al., 2008). First, the MSPSS assesses different sources of support (i.e., family, friends, and significant others). Second, this instrument is brief yet comprehensive, easy to understand. The MSPSS is a 12-item questionnaire, which is a selfadministered measure of perceived social support developed by Zimet et al. (1988). These authors found that the MSPSS had good reliability (with a Cronbach's alpha of 0.85 to 0.91 ). Furthermore, confirmatory factor analysis (CFA) showed a robust three-factor structure (family support, friend support, and significant-other support) of the MSPSS existed (Zimet et al., 1988). In addition, the MSPSS was found to be negatively related to parenting stress, loneliness and depression, and positively related to self-esteem and life satisfaction, indicating good concurrent validity of the MSPSS (Dunst et al., 1986; Bruwer et al., 2008; Jeong et al., 2013; Zhao et al., 2013; Kong et al., 2015).

Over the years, the MSPSS has been translated into many other languages such as Thai, Malaysian, Swedish, Polish, and demonstrated good reliability and validity (Duru, 2007; Ng et al., 2010; Wongpakaran and Wongpakaran, 2012; Adamczyk, 2013; Ekbäck et al., 2013). In addition, adequate psychometric properties of the MSPSS are also found in some special groups such as psychiatric patients, implantable cardioverter defibrillator patients, patients with heart failure (Cecil et al., 1995; Pedersen et al., 2009; Shumaker et al., 2017). All these results suggested that the MSPSS possessed good psychometric properties.

The Chinese MSPSS was first established by the Hong Kong scholar Chou (2000), and later the traditional Chinese version was translated into simplified Chinese language by Guan et al. (2015). These two studies confirmed the Chinese MSPSS had good reliability and validity. However, they only used adolescents and college students as a sample. In addition, the reliability and validity of the Chinese MSPSS have also been verified in patients with methadone maintenance treatment (Zhou et al., 2015). In recent years, the worldwide incidence of $\mathrm{CP}$ is still around $0.2 \%$ (Oliveira and Matsukura, 2013; Nelson and Blair, 2015). CP children's disorders in the development of posture and movement made the parents often experience higher levels of parenting stress (Ong et al., 1998), more chronic sorrow symptoms (Whittingham et al., 2013), and more irritable and anxious (Cheshire et al., 2010) than parents of normally developed children. Fortunately, there is evidence that social support has buffering effects on stressful life events and depression (Komatsu et al., 2010). Therefore, parents of children with CP need more social support to cope with stressful situations. However, parents of children with CP perceive low levels of social support than parents of children with normal development (Carona et al., 2013), parents of children with mentally retarded (Ashok, 2017), and rehabilitation professionals (Oliveira and Matsukura, 2013). There is no doubt that the social support of parents of children with CP is a matter of concern to researchers. A validated social support measurement tool for this particular group is a prerequisite for research. However, to our knowledge, the Chinese MSPSS has not been validated for the particular group of parents of children with CP. One purpose of this research was to examine the reliability, factorial validity and concurrent validity in relation to parenting stress of the Chinese MSPSS in parents of children with CP.
Another critical question when using the MSPSS is the measurement invariance across different gender groups. Prior studies have explored the difference in the MSPSS scores between fathers and mothers. For example, Ghorbani et al. (2014) found that the MSPSS scores of mothers of children with disabilities were lower than that of fathers. However, Simons et al. (2007) showed that there was no significant gender difference in the MSPSS scores. There may be a number of factors contributing to these differences, but before we discuss gender differences, we must first ensure that the MSPSS can measure the same latent factors across gender groups. Thus, another purpose of this research was to determine if the measurement structure underlying social support is equivalent across gender.

To address these issues, we firstly performed a CFA to test if the three-factor structure of the MSPSS can be replicated in Chinese parents of children with CP $(N=487)$. Secondly, we tested measurement invariance of MSPSS across gender groups by a multi-group CFA. Finally, we tested the concurrent validity of the Chinese version of the MSPSS in relation to parenting stress, which was selected due to their reliable negative relationship with social support (Jeong et al., 2013). We expected that perceived support from family, friends, and significant others will be inversely related to parenting stress.

\section{MATERIALS AND METHODS}

\section{Participants and Procedure}

We used the MSPSS and PSI-SF to measure social support and parenting stress respectively. The questionnaires were mailed to directors of medical institutes, children's rehabilitation centers, and special education schools in mainland China. The directors were responsible for sending to the parents, and the inclusion criteria were as follows: (1) the child's age was between 1 and 12 years and has been diagnosed with CP; (2) the parents were Chinese-speaking and could independently complete the questionnaires. All participants signed a written informed consent and were asked to complete a set of questionnaires anonymously and voluntarily. Only one parent in a same family was asked to fill in the questionnaires. In total, we distributed 520 questionnaires and received 487 (mother $=366$, father $=121)$ valid questionnaires for a recovery rate of $93.7 \%$. The mean age of the participated parents is 33.69 (standard deviation $=6.12$ ), age range is $21-55$ years. The current study was approved by the Research Ethics Board of the local university.

\section{Measures}

To measure social support, we used the Chinese version of the MSPSS. There are four items for each subscale of the Chinese MSPSS. A seven-point Likert scale is used ( 1 = very strongly disagree; $7=$ very strongly agree). The complete scale ranges from 12 to 84 . A higher score equates with higher social support. In this study, the Cronbach's alpha coefficients of the family support (Items 3, 4, 8, 11), friend support (Items 6, 7, 9, 12), and significant-other support (Items $1,2,5,10$ ) subscales were 0.88 , 0.89 , and 0.87 , respectively. 
To measure parenting stress, we used the Chinese version of the Parenting Stress Index-Short Form (PSI-SF). The PSI-SF is a 36-item questionnaire developed by Abidin and Wilfong (1989) to measure parenting stress. It includes three factors: parental distress (PD) (Items 1-12), parent-child dysfunctional interaction (PCDI) (Items 13-23, 34), and difficult children (DC) (Items 24-33, 35-36). The form applies a five-point Likert scale ( $1=$ strongly disagree; $5=$ strongly agree). A higher score equates with higher parenting stress. The Chinese version of the PSI-SF has good reliability and validity (Yeh et al., 2001). In this study, the Cronbach's alpha coefficients of the PSI-SF's three factors were $0.90,0.86$, and 0.84 , respectively.

\section{Data Analysis}

To test if the three-factor structure of the MSPSS can be replicated in Chinese parents of children with $\mathrm{CP}$, a CFA was firstly performed using Amos 23.0. Because the Chi-Square is considered very sensitive to the sample size, the following indices were used to evaluate model fit: comparative fit index (CFI), nonnormal fit index (NNFI, also known as Tucker-Lewis Index), root mean square error of approximation (RMSEA), and standardized root mean square residual (SRMR) ( $\mathrm{Hu}$ and Bentler, 1999). Accordingly, an acceptable model fit is indicated by a CFI of $>0.90$, a NNFI of $>0.90$, a RMSEA of $<0.08$, and a SRMR of $<0.08$. Values of CFI and NNFI above 0.95 (Hoyle, 1995), RMSEA and SRMR < 0.06 (Maccallum et al., 1996) represent an excellent model fit.

Then, the configural, metric, and scalar invariance were selected to examine the measurement invariance of Chinese MSPSS across gender groups by a multi-group CFA. The difference for CFI and RMSEA ( $\triangle \mathrm{CF}$ and $\triangle \mathrm{RMSEA}$ ) were used as indices for tests of invariance. $\triangle \mathrm{CF}$ and $\triangle \mathrm{RMSEA}$ of equal to or $<0.01$ were considered strong invariance (Cheung and Rensvold, 2002; Kong, 2017).

In addition, the relationships among perceived social support from family, friends, and significant others with parenting stress were examined by Pearson correlations. And $p<0.05$ was considered statistically significant.

\section{RESULTS}

\section{Confirmatory Factor Analysis}

The CFA analysis found that all the fit indices of the threefactor model meet their corresponding criteria very well, $\chi^{2}(51)=170.67, p<0.001, \mathrm{CFI}=0.969$, NNFI $=0.959$, $\mathrm{RMSEA}=0.069, \mathrm{SRMR}=0.037$. The factor loadings ranged from 0.70 to 0.92 . The model of structure of social support was showed in Figure 1.

\section{Measurement Invariance Configural Invariance}

Configural invariance was first conducted to assess if the Chinese MSPSS has a three-factor structure for the gender groups. The result showed that the configural invariance model (M1) fitted the data very well, CFI $=0.967$, RMSEA $=0.051$ (Table 1), and all factor loading were significant $(p<0.05)$.

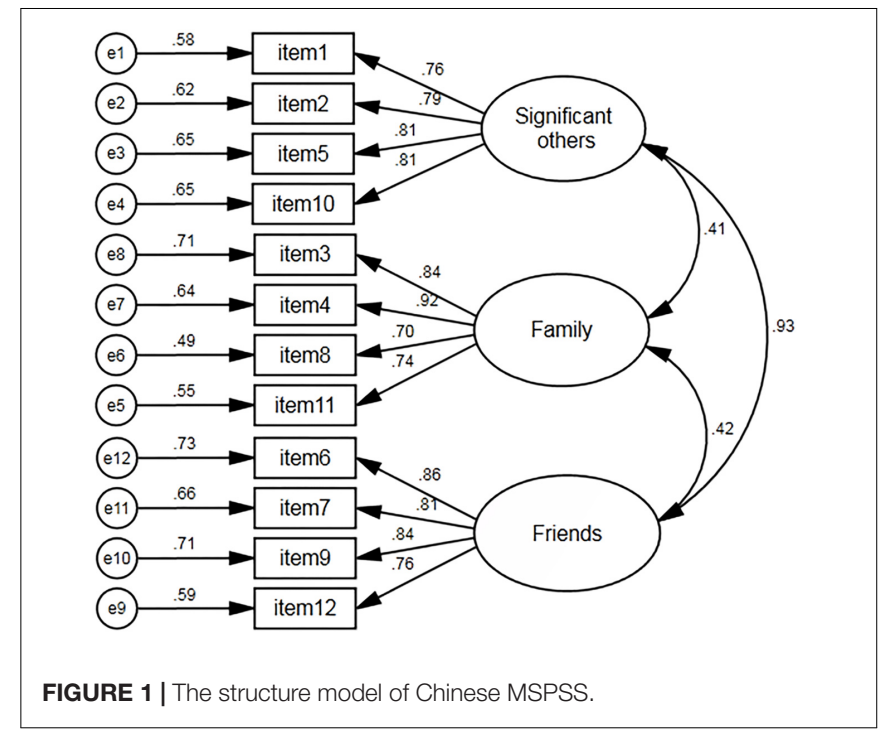

\section{Metric Invariance}

Next, a metric invariance model (M2) was conducted to verify the invariance of factor loadings across the two groups. The results showed a good fit, CFI $=0.965$, RMSEA $=0.050$ (Table 1). And no significant changes occurred when compared to the M1, $\Delta \mathrm{CFI}=0.002, \Delta \mathrm{RMSEA}=0.001$.

\section{Scalar Invariance}

Scalar invariance implies invariance of item intercepts across groups. Finally, scalar invariance model (M3) was established to test the intercepts and factor loadings were constrained to be equal across the fathers and mothers, and the residual variances were freely estimated. The results showed the model fitted well, CFI $=0.962$, RMSEA $=0.049$ (Table 1). And no significant changes occurred when compared to the M2, $\Delta \mathrm{CFI}=0.003$, $\triangle$ RMSEA $=0.001$.

Taking these results together, it is suggested that configural, metric, and scalar invariance hold across gender groups.

\section{Reliability}

Composite reliability was calculated as the internal consistency (Raykov, 1998) of the Chinese MSPSS. Compared to Cronbach's alpha reliability, it does not assume that all indicators are equally reliable. If the composite reliability is more than 0.7 , the model is good (Nunally, 1978). For all the parents, the composite reliability of the family support, friends support, and significant-other support subscales was $0.87,0.88$, and 0.89 , respectively. For the mothers, the composite reliability of these three subscales was $0.86,0.88$, and 0.89 , respectively. For the fathers, the composite reliability of these three subscales was $0.90,0.88$, and 0.90 , respectively. These results indicated that the internal consistency of the Chinese MSPSS was good.

\section{Descriptive Analysis and Concurrent Validity}

Concurrent validity is a supporting piece of evidence for construct validity. Pearson correlations were conducted with 
TABLE 1 | Fit indices for measurement invariance across gender.

\begin{tabular}{|c|c|c|c|c|c|c|c|c|c|}
\hline Model & $x^{2}$ & df & CFI & NNFI & RMSEA & SRMR & Comparison & $\Delta \mathrm{CFI}$ & $\Delta$ RMSEA \\
\hline M1 & 229.42 & 102 & 0.967 & 0.957 & 0.051 & 0.052 & & & \\
\hline M2 & 224.13 & 111 & 0.965 & 0.959 & 0.050 & 0.047 & M2 vs. M1 & 0.002 & 0.001 \\
\hline M3 & 267.68 & 123 & 0.962 & 0.959 & 0.049 & 0.048 & M3 vs. M2 & 0.003 & 0.001 \\
\hline
\end{tabular}

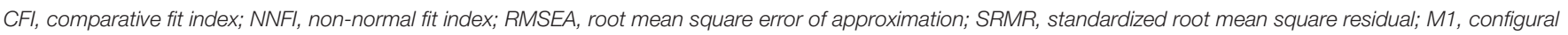
invariance model; M2, metric invariance model; M3, scalar invariance model.

TABLE 2 | Mean, SD, and correlation between the variables.

\begin{tabular}{|c|c|c|c|c|c|c|c|c|c|}
\hline & $M$ & $S D$ & 1 & 2 & 3 & 4 & 5 & 6 & 7 \\
\hline (1) Family & 20.4 & 6.11 & 1 & & & & & & \\
\hline (2) Friends & 17.1 & 6.23 & 0.515 & 1 & & & & & \\
\hline (3) Significant-other & 16.9 & 6.50 & 0.492 & 0.841 & 1 & & & & \\
\hline (4) Total MSPSS & 54.4 & 16.27 & 0.769 & 0.912 & 0.906 & 1 & & & \\
\hline (5) PD & 37.7 & 9.99 & -0.325 & -0.318 & -0.261 & -0.348 & 1 & & \\
\hline (6) PCDI & 29.2 & 8.25 & -0.179 & -0.212 & -0.198 & -0.227 & 0.526 & 1 & \\
\hline (7) $\mathrm{DC}$ & 33.6 & 8.47 & -0.156 & -0.167 & -0.173 & -0.192 & 0.443 & 0.691 & 1 \\
\hline (8) Total PSI & 100.4 & 22.31 & -0.271 & -0.284 & -0.256 & -0.313 & 0.810 & 0.868 & 0.833 \\
\hline
\end{tabular}

All correlations were significant at level of 0.01 . The numbers 1-7 of the first line of the table represents the same content as the vertical numbers.

the total sample to test the relationship among perceived social support from family, friends, and significant others with parenting stress to assess the concurrent validity between the Chinese version of MSPSS and PSI-SF subscales. The result showed that MSPSS was significant related with PSI-SF. The mean and standardized deviations of variables were presented in Table 2.

In addition, we examined the relationship between age factors and social support, and found that the correlation between children's age and social support was not significant (family: $r=-0.011, p=0.811$; friends: $r=-0.070, p=0.124$; significantothers: $r=-0.068, p=0.135$; total MSPSS: $r=-0.059$, $p=0.191$ ), and the correlation between parents' age and social support was not significant (family: $r=0.027, p=0.563$; friends: $r=-0.018, p=0.697$; significant-others: $r=-0.087, p=0.063$; total MSPSS: $r=-0.033, p=0.483$ ).

\section{DISCUSSION}

The present study aimed to investigate the reliability, factorial validity, measurement invariance across gender of the Chinese MSPSS in parents of children with CP. As we expected, the result showed that the three-factor structure was replicated in parents of children with CP. This is consistent with the original study by Zimet et al. (1988). In addition, the Chinese MSPSS had good internal consistency reliability with composite reliability values of more than 0.7. These results suggested that the Chinese MSPSS is a reliable and valid measure of social support perceived by parents of children with CP.

Importantly, the multi-group CFA demonstrated configural, metric, and scalar invariance of the Chinese MSPSS between mothers and fathers of children with CP. This is similar to the results of Cheng and Chan (2004) with the samples of adolescents. To our knowledge, this is the first report of measurement invariance across sex of the Chinese MSPSS in parents of children with CP. This finding indicates that the structure of Chinese MSPSS does measure the same construct for fathers and mothers of children with CP. Thus, in further research the fathers and mothers can be put together without worrying about gender effects on the structure of Chinese MSPSS.

Besides, the Pearson's correlation analysis revealed that the total MSPSS was significantly related to the total PSI-SF $(r=-0.313, p<0.01)$. This is consistent with the previous study on the relationship of MSPSS and PSI-SF for caregivers of children with developmental disabilities $(r=-0.390, p<0.01)$ (Peer, 2011). In addition, the three subscales of MSPSS were all significantly related to the three subscales of PSI-SF. The strong correlation between parenting stress and social support confirms that the Chinese version of the MSPSS has good concurrent validity.

\section{Strengths and Limitations}

In summary, the present findings suggest that the Chinese MSPSS displays adequate psychometric properties in terms of internal consistency, factor structure validity, and measurement invariance across gender in parents of children with $\mathrm{CP}$, so it can be applied to these particular populations to measure perceived social support. The availability of the MSPSS might facilitate the examination of the relationship and causal mechanism involved in the link between perceived social support and mental health among Chinese parents of children with CP. Although the present study yielded the expected results, there were still some limitations that should be noted. Firstly, the samples used in the current study are all Chinese, so we cannot be sure that the factor structure and measurement invariance of MSPSS can be obtained in other language versions. Secondly, the study used only cross- 
sectional data, so we can't confirm whether our results hold across different time periods.

\section{AUTHOR CONTRIBUTIONS}

All authors listed have made a substantial, direct and intellectual contribution to the work, and approved it for publication.

\section{REFERENCES}

Abidin, R. R., and Wilfong, E. (1989). Parenting stress and its relationship to child health care. Child Health Care 18, 114-116. doi: 10.1207/s15326888ch c1802_9

Adamczyk, K. (2013). Development and validation of the Polish-language version of the multidimensional scale of perceived social support (MSPSS). Rev. Int Psychol. Soc. 26, 25-48.

Ashok, R. (2017). Level of different family stress and influences of social support among parents of mentally retarded and cerebral palsy children. Int. J. Recent Sci. Res. 8, 18461-18466. doi: 10.24327/ijrsr.2017.0807.0513

Bruwer, B., Emsley, R., Kidd, M., Lochner, C., and Seedat, S. (2008). Psychometric properties of the multidimensional scale of perceived social support in youth. Compr. Psychiatry 49, 195-201. doi: 10.1016/j.comppsych.2007. 09.002

Campbell, P., Wynne-Jones, G., and Dunn, K. M. (2011). The influence of informal social support on risk and prognosis in spinal pain: a systematic review. Eur. J. Pain 15, 444. e1-444. e14.

Carona, C., Crespo, C., and Canavarro, M. C. (2013). Similarities amid the difference: caregiving burden and adaptation outcomes in dyads of parents and their children with and without cerebral palsy. Res. Dev. Disabil. 34, 882-893. doi: 10.1016/j.ridd.2012.12.004

Cecil, H., Stanley, M. A., Carrion, P. G., and Swann, A. (1995). Psychometric properties of the MSPSS and NOS in psychiatric outpatients. J. Clin. Psychol. 51, 593-602. doi: 10.1002/1097-4679(199509)51:5<593::AID-JCLP2270510503>3. $0 . \mathrm{CO} ; 2-\mathrm{W}$

Cheng, S.-T., and Chan, A. C. (2004). The multidimensional scale of perceived social support: dimensionality and age and gender differences in adolescents. Pers. Individ. Dif. 37, 1359-1369. doi: 10.1016/j.paid.2004.01.006

Cheshire, A., Barlow, J. H., and Powell, L. A. (2010). The psychosocial well-being of parents of children with cerebral palsy: a comparison study. Disabil. Rehabil. 32, 1673-1677. doi: 10.3109/09638281003649920

Cheung, G. W., and Rensvold, R. B. (2002). Evaluating goodness-of-fit indexes for testing measurement invariance. Struct. Equ. Modeling 9, 233-255. doi: 10.1207/S15328007SEM0902_5

Chou, K.-L. (2000). Assessing Chinese adolescents' social support: the multidimensional scale of perceived social support. Pers. Individ. Dif. 28, 299-307. doi: 10.1016/S0191-8869(99)00098-7

Dunst, C. J., Trivette, C. M., and Cross, A. H. (1986). Mediating influences of social support: personal, family, and child outcomes. Am. J. Ment. Defic. 90, 403-417.

Duru, E. (2007). Re-examination of the psychometric characteristics of the multidimensional scale of perceived social support among Turkish university students. Soc. Behav. Pers. 35, 443-452. doi: 10.2224/sbp.2007.35.4.443

Ekbäck, M., Benzein, E., Lindberg, M., and Årestedt, K. (2013). The Swedish version of the multidimensional scale of perceived social support (MSPSS)-a psychometric evaluation study in women with hirsutism and nursing students. Health Qual. Life Outcomes 11:168. doi: 10.1186/1477-7525-11-168

Ghorbani, M., Dolatian, M., Shams, J., and Alavi-Majd, H. (2014). Anxiety, posttraumatic stress disorder and social supports among parents of premature and full-term infants. Iran. Red Crescent Med. J. 16:e13461. doi: 10.5812/ircmj. 13461

Guan, N. C., Seng, L. H., Hway Ann, A. Y., and Hui, K. O. (2015). Factorial validity and reliability of the Malaysian simplified Chinese version of multidimensional scale of perceived social support (MSPSS-SCV) among a group of university students. Asia Pac. J. Public Health 27, 225-231. doi: 10. $1177 / 1010539513477684$

\section{ACKNOWLEDGMENTS}

We thank all the participants. This study was sponsored by Peak Discipline Construction Project of Education at East China Normal University; and the National Social Science Foundation Project in China (grant number 13AZD097) and the Fundamental Research Funds for the Central Universities (GK20170.090).

Hoyle, R. H. (1995). Structural equation modeling: concepts, issues, and applications. Libr. Q. 66, 253-256.

Hu, L. t., and Bentler, P. M. (1999). Cutoff criteria for fit indexes in covariance structure analysis: conventional criteria versus new alternatives. Struct. Equ. Modeling 6, 1-55. doi: 10.1080/10705519909540118

Jeong, Y.-G., Jeong, Y.-J., and Bang, J.-A. (2013). Effect of social support on parenting stress of Korean mothers of children with cerebral palsy. J. Phys. Ther. Sci. 25, 1339-1342. doi: 10.1589/jpts.25.1339

Kim, H. S., Sherman, D. K., and Taylor, S. E. (2008). Culture and social support. Am. Psychol. 63, 518-526 doi: 10.1037/0003-066X

Komatsu, Y., Kai, Y., Nagamatsu, T., Shiwa, T., Suyama, Y., and Sugimoto, M. (2010). Buffering effect of social support in the workplace on job strain and depressive symptoms. Sangyo Eiseigaku Zasshi 52, 140-148. doi: 10.1539/ sangyoeisei.B9012

Kong, F. (2017). The validity of the Wong and Law Emotional Intelligence Scale in a Chinese sample: tests of measurement invariance and latent mean differences across gender and age. Pers. Individ. Dif. 116, 29-31. doi: 10.1016/j.paid.2017. 04.025

Kong, F., Ding, K., and Zhao, J. (2015). The relationships among gratitude, selfesteem, social support and life satisfaction among undergraduate students. J. Happiness Stud. 16, 477-489. doi: 10.1007/s10902-014-9519-2

Maccallum, R. C., Browne, M. W., and Sugawara, H. M. (1996). Power analysis and determination of sample size for covariance structure modeling. Psychol. Methods 1, 130-149. doi: 10.1037/1082-989X.1.2.130

Nelson, K. B., and Blair, E. (2015). Prenatal factors in singletons with cerebral palsy born at or near term. N. Engl. J. Med. 373, 946-953. doi: 10.1056/ NEJMra1505261

Ng, C. G., Siddiq, A. N. A., Aida, S. A., Zainal, N. Z., and Koh, O. H. (2010). Validation of the Malay version of the multidimensional scale of perceived social support (MSPSS-M) among a group of medical students in faculty of medicine, University Malaya. Asian J. Psychiatry 3, 3-6. doi: 10.1016/j.ajp.2009. 12.001

Nunally, J. C. (1978). Psychometric Theory, 2nd Edn. New York, NY: McGraw-Hill. Oliveira, A. K. C. D., and Matsukura, T. S. (2013). Stress and social support in caregivers of children with cerebral palsy. Cad. Ter. Occup. 21, 493-503.

Ong, L. C., Afifah, I., Sofiah, A., and Lye, M. S. (1998). Parenting stress among mothers of Malaysian children with cerebral palsy: predictors of child- and parent-related stress. Ann. Trop. Paediatr. 18, 301-307. doi: 10.1080/02724936. 1998.11747964

Pedersen, S. S., Spinder, H., Erdman, R. A., and Denollet, J. (2009). Poor perceived social support in implantable cardioverter defibrillator (ICD) patients and their partners: cross-validation of the multidimensional scale of perceived social support. Psychosomatics 50, 461-467. doi: 10.1016/S0033-3182(09)70838-2

Peer, J. W. (2011). Coping Style as a Mediator of Stress Perception for Caregivers of Children with Developmental Disabilities. Ph.D. dissertations, Wayne State University, Detroit, MI.

Raykov, T. (1998). Coefficient alpha and composite reliability with interrelated nonhomogeneous items. Appl. Psychol. Meas. 22, 375-385. doi: 10.1177/ 014662169802200407

Shumaker, S. C., Frazier, S. K., Moser, D. K., and Chung, M. L. (2017). Psychometric properties of the multidimensional scale of perceived social support in patients with heart failure. J. Nurs. Meas. 25, 90-102. doi: 10.1891/1061-3749. 25.1.90

Simons, L., Ingerski, L. M., and Janicke, D. M. (2007). Social support, coping, and psychological distress in mothers and fathers of pediatric transplant candidates: 
a pilot study. Pediatr. Transplant. 11, 781-787. doi: 10.1111/j.1399-3046.2007. 00726.x

Stanley, M. A., Beck, J. G., and Zebb, B. J. (1998). Psychometric properties of the MSPSS in older adults. Aging Ment. Health 2, 186-193. doi: 10.1080/ 13607869856669

Whittingham, K., Wee, D., Sanders, M., and Boyd, R. (2013). Predictors of psychological adjustment, experienced parenting burden and chronic sorrow symptoms in parents of children with cerebral palsy. Child Care Health Dev. 39, 366-373. doi: 10.1111/j.1365-2214.2012.01396.x

Wongpakaran, N., and Wongpakaran, T. (2012). A revised thai multi-dimensional scale of perceived social support. Span. J. Psychol. 15, 1503-1509. doi: 10.5209/ rev_SJOP.2012.v15.n3.39434

Yeh, C. H., Chen, M. L., Li, W., and Chuang, H. L. (2001). The Chinese version of the parenting stress index: a psychometric study. Acta Paediatr. 90, 1470-1477. doi: 10.1111/j.1651-2227.2001.tb01615.x

Zhao, J., Kong, F., and Wang, Y. (2013). The role of social support and selfesteem in the relationship between shyness and loneliness. Pers. Individ. Dif. 54, 577-581. doi: 10.1016/j.paid.2012.11.003
Zhou, K., Li, H., Wei, X., Yin, J., Liang, P., Zhang, H., et al. (2015). Reliability and validity of the multidimensional scale of perceived social support in Chinese mainland patients with methadone maintenance treatment. Compr. Psychiatry 60, 182-188. doi: 10.1016/j.comppsych.2015.03.007

Zimet, G. D., Dahlem, N. W., Zimet, S. G., and Farley, G. K. (1988). The multidimensional scale of perceived social support. J. Pers. Assess. 52, 30-41. doi: 10.1207/s15327752jpa5201_2

Conflict of Interest Statement: The authors declare that the research was conducted in the absence of any commercial or financial relationships that could be construed as a potential conflict of interest.

Copyright (c) 2017 Wang, Wan, Huang, Huang and Kong. This is an open-access article distributed under the terms of the Creative Commons Attribution License (CC BY). The use, distribution or reproduction in other forums is permitted, provided the original author(s) or licensor are credited and that the original publication in this journal is cited, in accordance with accepted academic practice. No use, distribution or reproduction is permitted which does not comply with these terms. 